\title{
From the ashes: JVLA observations of water fountain nebula candidates show the rebirth of IRAS 18455+0448
}

\author{
W. H. T. Vlemmings ${ }^{1}$, N. Amiri ${ }^{2}$, H. J. van Langevelde ${ }^{3,4}$, and D. Tafoya ${ }^{5}$ \\ ${ }^{1}$ Department of Earth and Space Sciences, Chalmers University of Technology, Onsala Space Observatory, 43992 Onsala, Sweden \\ e-mail: wouter.vlemmings@chalmers.se \\ 2 Center for Astrophysics and Space Astronomy, Department of Astrophysical and Planetary Sciences, University of Colorado, \\ 389 UCB, Boulder, CO, 80309-0389, USA \\ 3 Joint Institute for VLBI in Europe, Postbus 2, 7990 AA Dwingeloo, The Netherlands \\ ${ }^{4}$ Sterrewacht Leiden, Leiden University, Postbus 9513, 2300 RA Leiden, The Netherlands \\ 5 Centro de Radioastronomía y Astrofísica, UNAM, Apdo. Postal 3-72 (Xangari), 58089 Morelia, Michoacán, México
}

Received 4 March 2014 / Accepted 24 July 2014

\begin{abstract}
Context. The class of water fountain nebulae is thought to represent the stage of the earliest onset of collimated bipolar outflows during the post-asymptotic giant branch phase. They thus play a crucial role in the study of the formation of bipolar planetary nebulae (PNe). To date, 14 water fountain nebulae have been identified. The identification of more sources in this unique stage of stellar evolution will enable us to study the origin of bipolar PNe morphologies in more detail.

Aims. Water fountain candidates can be identified based on the often double peaked $22 \mathrm{GHz} \mathrm{H}_{2} \mathrm{O}$ maser spectrum with a large separation between the maser peaks (often $>100 \mathrm{~km} \mathrm{~s}^{-1}$ ). However, even a fast bipolar outflow will only have a moderate velocity extent in its maser spectrum when located close to the plane of the sky. In this project we aim to enhance the water fountain sample by identifying objects whose jets are aligned close to the plane of the sky.

Methods. We present the results of seven sources observed with the Jansky Very Large Array (JVLA) that were identified as water fountain candidates in an Effelsberg $100 \mathrm{~m}$ telescope survey of 74 AGB and early post-AGB stars.

Results. We find that our sample of water fountain candidates displays strong variability in their $22 \mathrm{GHz} \mathrm{H}_{2} \mathrm{O}$ maser spectra. The JVLA observations show an extended bipolar $\mathrm{H}_{2} \mathrm{O}$ maser outflow for one source, the OH/IR star IRAS 18455+0448. This source was previously classified as a dying $\mathrm{OH} / \mathrm{IR}$ star based on the exponential decrease of its $1612 \mathrm{MHz} \mathrm{OH}$ maser and the lack of $\mathrm{H}_{2} \mathrm{O}$ masers. We therefore also re-observed the 1612,1665 , and $1667 \mathrm{MHz} \mathrm{OH}$ masers. We confirm that the $1612 \mathrm{MHz}$ masers have not reappeared and find that the $1665 / 1667 \mathrm{MHz}$ masers have decreased in strength by several orders of magnitude during the last decade. The JVLA observations also reveal a striking asymmetry in the red-shifted maser emission of IRAS 19422+3506.

Conclusions. The OH/IR star IRAS $18455+0448$ is confirmed to be a new addition to the class of water fountain nebulae. Its kinematic age is $\sim 70 \mathrm{yr}$, but could be lower, depending on the distance and inclination. Previous observations indicate, with significant uncertainty, that IRAS $18455+0448$ has a surprisingly low mass compared to available estimates for other water fountain nebulae. The available historical $\mathrm{OH}$ maser observations make IRAS $18455+0448$ unique for the study of water fountain nebulae and the launch of post-AGB bipolar outflows. The other candidate sources appear high mass-loss OH/IR stars with partly radially beamed $\mathrm{H}_{2} \mathrm{O}$ masers.
\end{abstract}

Key words. stars: AGB and post-AGB - masers

\section{Introduction}

The origin of the bipolar morphologies observed in a large number of young planetary nebulae (PNe) and post-asymptotic giant branch (AGB)/pre-PNe sources is a long-standing question. Various mechanisms explaining the observed morphologies have been invoked (Balick \& Frank 2002). Of these, collimated outflows launched during the late AGB or early post-AGB phase has been suggested as the most direct explanation for the formation of bipolar PNe (e.g., Sahai \& Trauger 1998). The origin of these collimated outflows, or bipolar jets, is however still unclear. The collimation of an outflow due to a magnetic field, similar to what is observed for proto-stars, is a promising candidate, even though such a field might require a binary companion or massive planet to be maintained sufficiently long (e.g., Garcia-Segura et al. 2014). Alternative common-envelope evolutionary scenarios in which a binary companion becomes engulfed by the expanding AGB star could also provide an explanation (e.g., Nordhaus et al. 2007).

In order to constrain the launching mechanisms of bipolar outflows in late-type stars, it is important to find the youngest bipolar sources where the ejection of material has begun just recently. These are often still in, or just after, the AGB phase of their evolution. For these sources it can be possible to study simultaneously both the bipolar outflows as well as the remnants of their AGB envelopes. The youngest of the bipolar pre$\mathrm{PNe} /$ post-AGB stars are the class of water fountain sources. These display $\mathrm{H}_{2} \mathrm{O}$ maser emission at velocities that can reach up to several hundred $\mathrm{km} \mathrm{s}^{-1}$. This is significantly beyond that of the regularly expanding envelopes that can sometimes still be traced in $\mathrm{OH}$ masers, with typical expansion velocities of $\sim 15 \mathrm{~km} \mathrm{~s}^{-1}$ (Likkel et al. 1992). Interferometric observations of these sources have revealed that they posses tightly collimated jets that excite $\mathrm{H}_{2} \mathrm{O}$ masers at the shocked interface ahead or 
Table 1. Observed sources.

\begin{tabular}{lcccccccc}
\hline \hline Source & $\begin{array}{c}\alpha_{\mathrm{J} 2000} \\
\mathrm{hh} \mathrm{mm} \mathrm{ss}\end{array}$ & $\begin{array}{c}\delta_{\mathrm{J} 2000} \\
{ }^{\prime}{ }^{\prime \prime}\end{array}$ & $\begin{array}{c}D^{a} \\
\mathrm{kpc}\end{array}$ & $\begin{array}{c}V_{\mathrm{lsr}}^{b} \\
\mathrm{~km} \mathrm{~s}^{-1}\end{array}$ & $\begin{array}{c}\Delta V_{\mathrm{OH}} \\
\mathrm{km} \mathrm{s}^{-1}\end{array}$ & $\begin{array}{c}\Delta V_{\mathrm{H}_{2} \mathrm{O}}^{c} \\
\mathrm{~km} \mathrm{~s}^{-1}\end{array}$ & $\begin{array}{c}\Delta_{\text {red-blue }} \\
\text { mas }\end{array}$ & Refs. \\
\hline IRAS 18455+0448 & 184802.30 & +045130.446 & $2.1 / 11.2$ & 34.1 & 12.5 & 38.7 & $49 \pm 15$ & 1 \\
IRAS 19067+0811 & 190908.31 & +081633.802 & $3.7 / 8.6$ & $62.8 / 59.2$ & 32.5 & 31.8 & $13 \pm 12$ & 2,3 \\
IRAS 19069+0916 & 190919.25 & +092111.529 & $2.0 / 10.1$ & $30.0 / 31.7$ & 43.6 & 38.1 & $8 \pm 6$ & 4,5 \\
IRAS 19112+0819 & 191337.32 & +082452.489 & $2.4 / 9.8$ & 38.6 & 41.8 & 33.6 & $18 \pm 45$ & 6 \\
IRAS 19186+0315 & 192111.71 & +032057.800 & 14.7 & $-23.8 /-22.0$ & 31.8 & 28.0 & - & 7,5 \\
IRAS 19254+1631 & 192742.03 & +163724.182 & 10.3 & $3.1 / 1.9$ & 37.2 & 29.0 & $19 \pm 15$ & 3,8 \\
IRAS 19422+3506 & 194407.00 & +351408.207 & 10.3 & $-48.8 /-48.4$ & 34.0 & 34.7 & $4 \pm 5$ & 7,9 \\
\hline
\end{tabular}

Notes. ${ }^{(a)}$ Near/far kinematic distance (using Reid et al. 2009). ${ }^{(b)}$ Literature averaged $\mathrm{SiO}$ and OH systemic velocities. If both are the same or SiO is unavailable only a single value is given. ${ }^{(c)}$ Based on the JVLA observations, except for IRAS 19186+0315, for which the third Effelsberg epoch was used;

References. 1 Lewis et al. (2001); 2 Kim et al. (2010); 3 Engels et al. (1986); 4 Nakashima \& Deguchi (2003); 5 Eder et al. (1988); 6 Chengalur et al. (1993); 7 Kim et al. (2013); 8 te Lintel Hekkert et al. (1989); 9 Lewis (1997).

around them (Imai 2007). These jets have kinematic ages of only a few tens to hundreds of years and synchrotron and maser polarization observations indicate that at least a few of them are collimated by a strong magnetic field (Vlemmings et al. 2006; Pérez-Sánchez et al. 2013). The Galactic scale height of the water fountain population, the heavy circumstellar extinction and observations of ${ }^{12} \mathrm{CO}$ and ${ }^{13} \mathrm{CO}$ appears to indicate that their progenitor stars are fairly massive ( $>4 M_{\odot}$ He et al. 2008; Suárez et al. 2008; Imai et al. 2012; Rizzo et al. 2013). None of the water fountains has had a binary companion directly confirmed, although jet-precession in some of them could be due to an embedded companion (Imai 2007; Yung et al. 2011).

Only 14 water fountains are known to date. Since their detection is often related to the observations of $\mathrm{H}_{2} \mathrm{O}$ masers spread over a very large velocity range, detection is necessarily biased towards bipolar sources closest to the line of sight. In order to identify water fountain nebulae whose jets lie close to the plane of the sky, a combination of single dish monitoring and interferometric observations is required. Here we present JVLA and Effelsberg observations of a number of candidate water fountain sources with a smaller spread of their $\mathrm{H}_{2} \mathrm{O}$ masers. The source sample, observations and data reduction are presented in Sect. 2. The resulting maser spectra and $\mathrm{H}_{2} \mathrm{O}$ maser variability are described in Sect. 3, and in particular the results of the confirmed water fountain IRAS 18455+0448 are discussed in Sect. 4 .

\section{Observations}

\subsection{The sample}

The sources in our sample were originally observed as part of the observational campaign presented and discussed in Amiri (2011). In that work, a sample of 74 late AGB or early post-AGB objects was observed in an attempt to detect their $\mathrm{H}_{2} \mathrm{O}$ maser emission. In this work, we focused on seven sources that were either newly discovered or have distinct double or multipeaked $\mathrm{H}_{2} \mathrm{O}$ maser emission with a velocity spread close to that of the $\mathrm{OH}$ masers. The sources are listed in Table 1. One of the sources identified in Amiri (2011) as a water fountain candidate,IRAS $18455+0448$, was also proposed as a water fountain based on later Effelsberg observations by Yung et al. (2013). As earlier observations indicated an exponential decline in the $1612 \mathrm{MHz} \mathrm{OH}$ maser line for this source (Lewis et al. 2001), we also performed new 1612, 1665, and $1667 \mathrm{MHz} \mathrm{OH}$ maser observations for IRAS $18455+0448$.

\subsection{Effelsberg observations}

Observations of the $22.23508 \mathrm{GHz} \mathrm{H}_{2} \mathrm{O}$ masers in our sample were done with the Effelsberg telescope over three epochs: 12 and 13 November 2009, 22 and 23 March 2011, and 14 April 2011. For the first two epochs we used the $1.3 \mathrm{~cm}$ prime focus receiver in spectral line mode. The $1.3 \mathrm{~cm}$ focus secondary VLBI receiver was used for the third epoch observations.

The full width half maximum (FWHM) of the Effelsberg telescope is 40.2 at the maser frequency. Using the FFT spectrometer with 16384 channels and a bandwidth of $100 \mathrm{MHz}$, equivalent to a velocity coverage of $1363 \mathrm{~km} \mathrm{~s}^{-1}$, centered at the stellar velocity, the resulting spectral resolution is $0.08 \mathrm{~km} \mathrm{~s}^{-1}$. The observations were made in position switching mode with a cycle of $1 \mathrm{~min}$, sufficient to compensate for atmospheric fluctuations. The observing time for each source was 8-16 min at epoch 1, $8 \mathrm{~min}$ at epoch 2 and $12-24 \mathrm{~min}$ at epoch 3 . The rms noise for the first, second and third epochs corresponds to $0.02-0.18,0.06$ and $0.07-0.3 \mathrm{Jy}$, respectively.

For the $\mathrm{OH}$ maser observations of IRAS $18455+0448$, we used the $21 / 18 \mathrm{~cm}$ primary focus receiver. At the $\mathrm{OH}$ maser frequency the FWHM of the telescope corresponds to $\sim 470$ arcsec. Mainline $\mathrm{OH}$ maser observations at 1665.4018 and 1667.359 were performed on 22 Feb. 2010 with a bandwidth of $20 \mathrm{MHz}$ and 16384 channels which provides a channel spacing of $\sim 0.2 \mathrm{~km} \mathrm{~s}^{-1}$. The total on source observing time was $2 \mathrm{~h}$ and $26 \mathrm{~min}$. The observations of $1612 \mathrm{MHz}$ masers were made on 27 March 2010 with a bandwidth of $100 \mathrm{MHz}$ and 16384 channels corresponding to a channel spacing of $\sim 1 \mathrm{~km} \mathrm{~s}^{-1}$. The total on source observing time was $72 \mathrm{~min}$. The rms noise in channels free of emission is $\sim 7 \mathrm{mJy}$ for both epochs.

The data reduction was performed using the Continuum and Line Analysis Single-dish Software (CLASS) that is part of the GILDAS package ${ }^{1}$. We obtained the raw spectra in units of temperature counts. The spectra were converted to units of antenna temperature $\left(T_{\mathrm{A}}\right)$ by applying the calibration noise temperature. We also corrected the spectra for atmospheric opacity and the gain-elevation effect, the measurements of which were provided by the observatory. Finally, the spectra were converted to Jy units by dividing the spectra by the sensitivity of the telescope at the maser frequency.

1 http://www.iram.fr/IRAMFR/GILDAS 

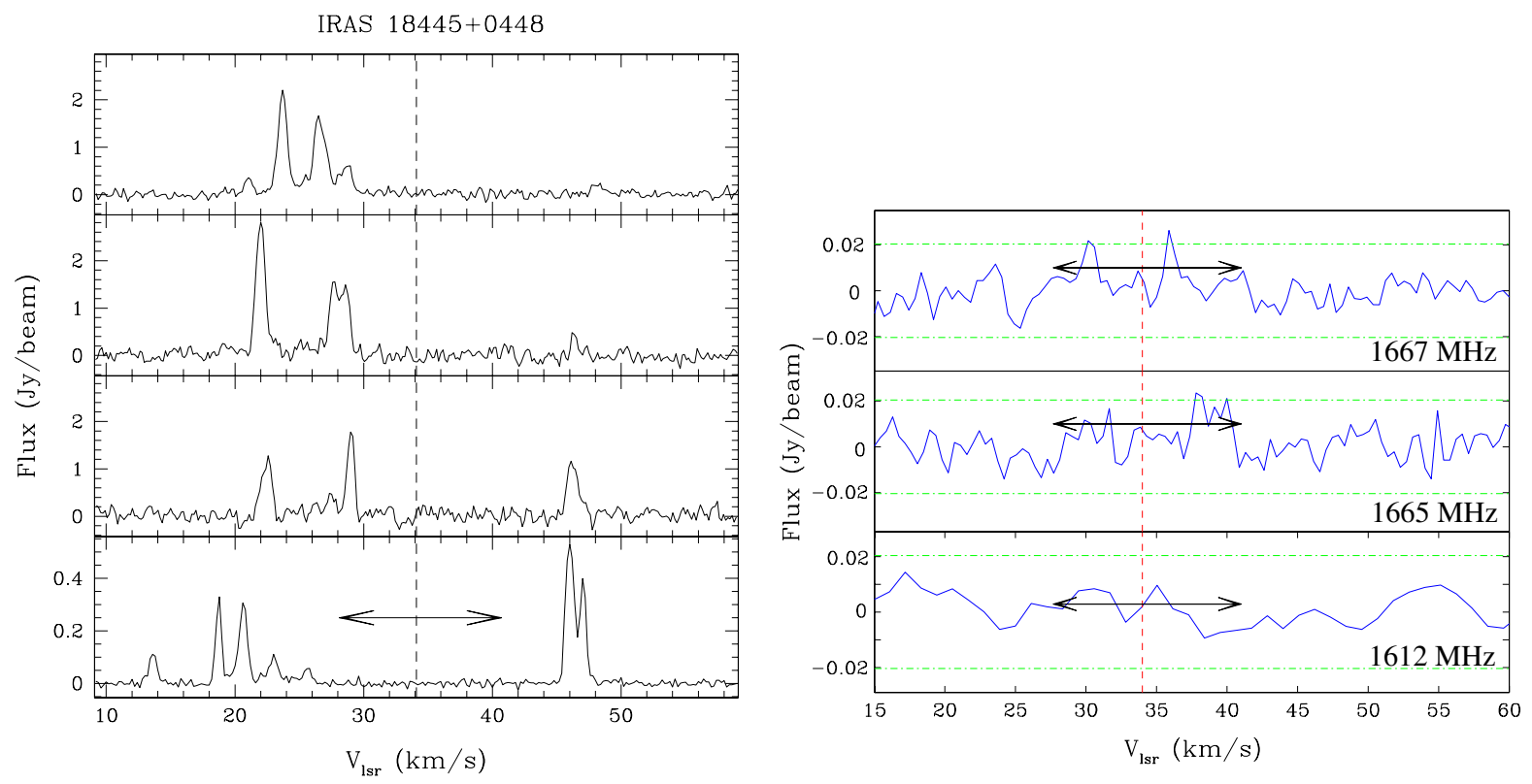

Fig. 1. Left: spectra of the $\mathrm{H}_{2} \mathrm{O}$ masers of IRAS $18455+0448$ observed with the Effelsberg $100 \mathrm{~m}$ telescope (top three panels) and with the JVLA (bottom panel) at four different epochs (with different flux scales). From top to bottom, the epochs are 12-13 November 2009, 22-23 March 2011, 14 April 2011 and 6 June 2013. The arrows in the bottom panel indicate the spread of the previously observed $\mathrm{OH}$ masers and the vertical dashed line indicates the systemic velocity based on $\mathrm{SiO}$ maser observations. Right: confirmation of the disappearance of the $\mathrm{OH}$ masers at 1612,1665 and $1667 \mathrm{MHz}$. The masers were observed with Effelsberg at 22 February 2010 (1665 and $1667 \mathrm{MHz})$ and $27 \mathrm{March} 2010$ (1612 MHz). The vertical dashed line indicated the systemic velocity and the horizontal dotted-dashed lines the $3 \sigma$ noise limits. The arrows indicate the spread of $\mathrm{OH}$ masers observed by Lewis et al. (2001).

\subsection{JVLA observations}

In order to improve the positional accuracy of our sources for later VLBI observations and investigate the location and extent of the $\mathrm{H}_{2} \mathrm{O}$ masers, our sources were observed with the JVLA in C-configuration on 6 June 2013 (program 13A-088). We used a total bandwidth of $32 \mathrm{MHz}$ centered at the $\mathrm{H}_{2} \mathrm{O}$ maser rest frequency in dual polarization. The original 8192 channels were averaged to produce a data set with 4096 channels of $\sim 0.1 \mathrm{~km} \mathrm{~s}^{-1}$ width. The seven sources were observed interleaved with four phase calibrators $(\mathrm{J} 2007+4029$, J1851+0035, $\mathrm{J} 1922+1530$, and J1925+2106) during a total observing time of 3 h. Bandpass calibration was done using J2007+4029 and we performed periodic pointing observations at $X$-band.

The data calibration was performed using the Common Astronomy Software Application (CASA). As no suitable primary flux calibrator was visible during our short observing run, we rely on archive fluxes of our phase calibrators to determine the absolute flux of our sources. Based on this we conclude that our fluxes are uncertain at the level of $25 \%$. Although the positions of the masers in our sample were determined by directly fitting the uv-data, we also produced images and spectra further averaging to $0.2 \mathrm{~km} \mathrm{~s}^{-1}$ channels. The typical rms in our spectra is $7.2 \mathrm{mJy} /$ beam and the typical beamwidth is $\sim 1.2^{\prime \prime} \times 1.1^{\prime \prime}$. A direct comparison between the fluxes obtained from singledish Effelsberg and from interferometric JVLA observations is possible, since $\mathrm{H}_{2} \mathrm{O}$ masers with typical sizes of only a few milliarcseconds are too compact to be resolved out using the JVLA in C-configuration.

\section{Results}

\section{1. $\mathrm{H}_{2} \mathrm{O}$ maser spectra}

The Effelsberg and JVLA $\mathrm{H}_{2} \mathrm{O}$ maser spectra for IRAS $18455+0448$ and those for the other six sources are shown in Fig. 1 (left) and Fig. 2 respectively. All seven sources display significant variability in their $\mathrm{H}_{2} \mathrm{O}$ maser emission between the four epochs. In particular, we find strong variability for IRAS $19067+0811$ and IRAS 19112+0819, which were undetected in our first Effelsberg epoch, while for IRAS 19254+1631 only the JVLA observations reveal clear blue-shifted emission. Moreover, IRAS 19069+0916 and IRAS 19422+3506, display variability of almost two orders of magnitude between different epochs. The strong variability could also be the cause of the initial non-detection of IRAS 18455+0448 (see however Sect. 4.1), IRAS 19112+0819 and IRAS 19254+1631 in earlier observations by Engels \& Lewis (1996).

In all cases except IRAS $18455+0448$, the $\mathrm{H}_{2} \mathrm{O}$ maser velocity spread is similar to that of the $\mathrm{OH}$ masers. While all of the sources display at least a prominent double peaked spectrum, IRAS 18455+0448, IRAS 19254+1631 and IRAS 19422+3506 also display several other strong maser features across their spectrum.

\section{2. $\mathrm{OH}$ masers of IRAS $18455+0448$}

The OH maser spectra of IRAS $18455+0448$ are shown in Fig. 1 (right). The $\mathrm{OH} 1612 \mathrm{MHz}$ maser is not detected after being measured to have a peak intensity of $\sim 40 \mathrm{mJy}$ in 2000 (Lewis et al. 2001). In that epoch, the 1665 and $1667 \mathrm{MHz}$ OH masers were observed to have peak fluxes of $\sim 450 \mathrm{mJy}$ and $\sim 730 \mathrm{mJy}$ respectively. In our current observations we only tentatively detect possible peaks of the 1665 and $1667 \mathrm{MHz} \mathrm{OH}$ masers at $25 \pm 7 \mathrm{mJy}$ (at $V_{\mathrm{lsr}} \approx 38 \mathrm{~km} \mathrm{~s}^{-1}$ ) and $22 \pm 7 \mathrm{mJy}$ (at $V_{\text {lsr }} \approx 30$ and $36 \mathrm{~km} \mathrm{~s}^{-1}$ ) respectively. The relative velocity, with respect to the star, of these potential maser peaks are less than what was measured in 2000 as indicated in Fig. 1 (right) by the arrow. The main-line masers have thus also faded by more than an order of magnitude over the last decade. 
IRAS $19067+0811$

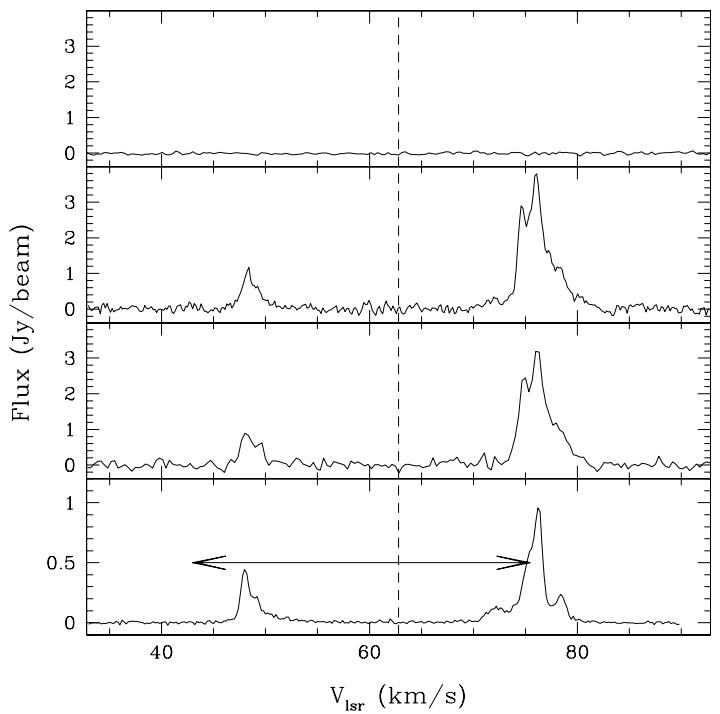

IRAS 19112+0819

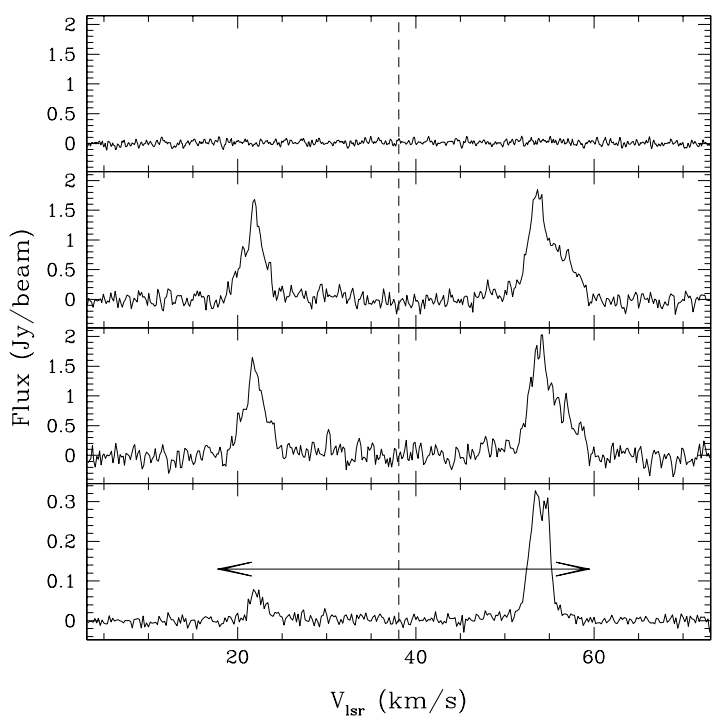

IRAS $19254+1631$

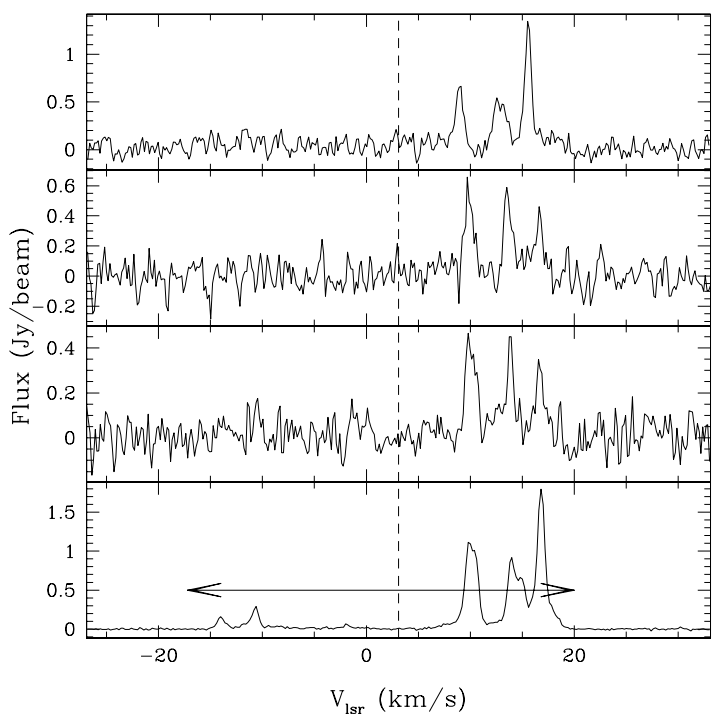

IRAS 19069+0916

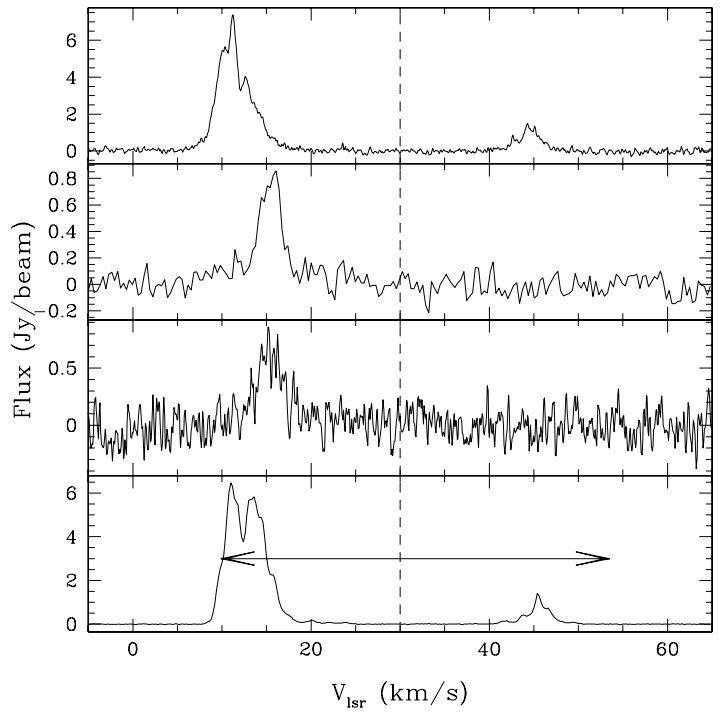

IRAS $19186+0315$

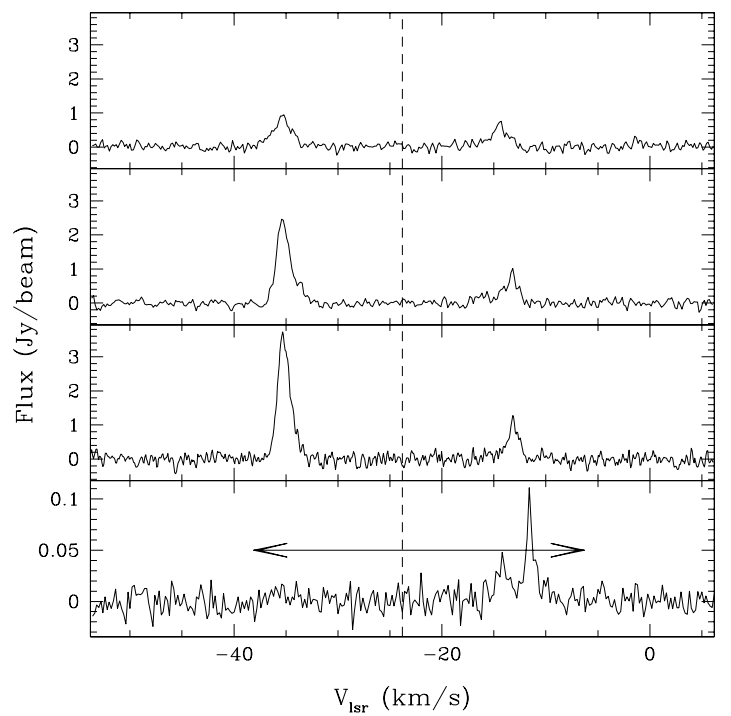

IRAS $19422+3506$

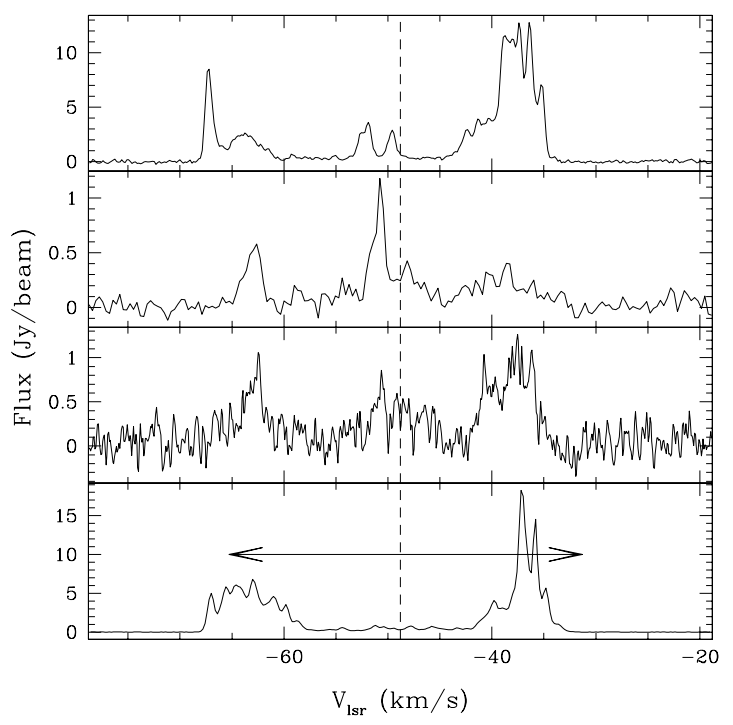

Fig. 2. Same as Fig. 1 (left) for the other six sources in our sample. We note the difference in flux scale for several of the observational epochs. The vertical dashed line indicates, where available, the literature averaged $\mathrm{SiO}$ maser velocity, otherwise the systemic velocity derived from the $\mathrm{OH}$ masers is used (see Table 1). 


\subsection{JVLA observations of the $\mathrm{H}_{2} \mathrm{O}$ maser components}

Our observations with the JVLA allowed us to determine more accurate positions and to investigate the offset between the redand blue-shifted masers. Although the $\sim 1^{\prime \prime}$ JVLA resolution is not sufficient to directly resolve the individual components, the strength of the masers allows us to reach positional accuracies of a few to a few tens of milliarcseconds for the maser positions (considering $\theta \approx$ Beam $/(2 \times S / N)$ ), where $\mathrm{S} / \mathrm{N}$ is the signal-to-noise ratio. We determined the maser positions by performing fits in the $(u, v)$-plane for each velocity channel assuming a single, unresolved, component (using the CASA code UVMULTIFIT, Martí-Vidal et al. 2014). Although in the spectra several individual features can be identified, these likely still consist of individual maser components blended in velocity. However, fitting multiple maser components within the relatively large JVLA beam would lead to poorly defined fits. The position errors determined from the $u, v$-fitting of a single component per velocity channel will, besides the aforementioned dependence on $\mathrm{S} / \mathrm{N}$ and beam-size, contain the uncertainty related to the potential existence of multiple components in the beam. Component maps for IRAS $18455+0448$ and IRAS 19422+3506 are shown in Figs. 3 and 4 respectively. The remaining sources are shown in Fig. 5. For all sources (except IRAS 19186+0315 where only red-shifted emission was detected), we determined the offset between the red- and blue-shifted emission by determining a $\mathrm{S} / \mathrm{N}$ weighted average of the channel component positions. The results of this analysis are presented in Table 1. That same table lists the JVLA observed spread of the $\mathrm{H}_{2} \mathrm{O}$ masers velocities $\left(\Delta V_{\mathrm{H}_{2} \mathrm{O}}\right)$ and the centroid positions determined by $\mathrm{S} / \mathrm{N}$ weighted averaging of all maser channels. As can be seen in Fig. 3, IRAS $18455+0448$ is the only source for which a significant off-set, of $49 \pm 15$ mas, is measured.

\section{Discussion}

\subsection{Nature of IRAS $18455+0448$}

IRAS $18455+0448$ is the only source of the sample for which a clear separation is seen between the red- and blue-shifted masers (Fig. 3) and for which the $\mathrm{H}_{2} \mathrm{O}$ maser velocity spread is more than twice that of the $\mathrm{OH}$ masers. It was also one of the three sources in our Effelsberg survey that displayed $\mathrm{H}_{2} \mathrm{O}$ masers that were not detected in the previous survey by Engels \& Lewis (1996) who reported an rms level of 0.2 Jy in 1990.

Based on the exponential fading of the $1612 \mathrm{MHz} \mathrm{OH}$ masers between 1988 and 2000, Lewis et al. (2001) suggested the source as the prototype of a "dying" OH/IR star. In their preferred model, the mass loss has gradually declined to close to 0 . Considering the dust velocity $\left(V_{\mathrm{e}, \mathrm{dust}}\right)$ is larger than that of the gas $\left(V_{e, g a s}\right)$, the expansion of both will differ. This results in a gradual decrease in the dust column density that shields the $\mathrm{OH}$ from the interstellar UV. The $\mathrm{OH}$ will thus be photo-dissociated more rapidly, thereby decreasing the column density in the maser region. This effect will become apparent after a time that is proportional to the expansion timescale from the photosphere to the $\mathrm{OH}$ maser region and the ratio $V_{\mathrm{e}, \mathrm{gas}} / V_{\mathrm{e} \text {,dust }}$. This is on the order of $1000 \mathrm{yr}$ for IRAS $18455+0448$. As the satellite $\mathrm{OH}$ line has the strongest dependence on column density, it will initially be affected before the main line masers. In the framework of the above model, an abrupt termination of the mass loss would not produce the exponential decline in maser flux, nor would it affect the $1612 \mathrm{MHz}$ masers before the main line masers. Thus, the model described by Lewis et al. (2001) is supported by the

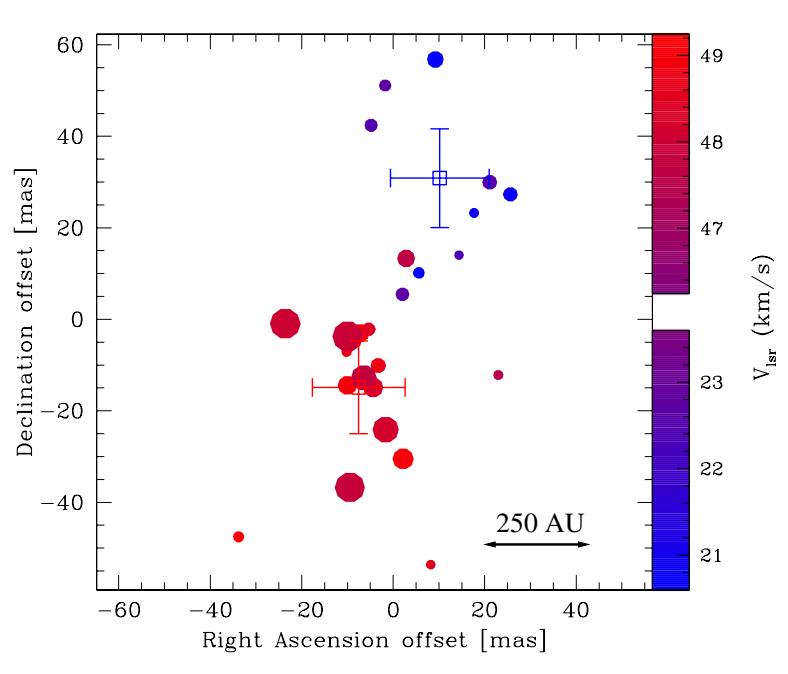

Fig. 3. The $\mathrm{H}_{2} \mathrm{O}$ maser distribution around IRAS $18455+0448$ observed with the JVLA. Fits to the centroid position of individual maser velocity channels are potted scaled based on their flux and color coded according to velocity. Only masers detected at a signal-to-noise-ratio $>15$ are plotted. The positional uncertainties on the individual symbols range from $\sim 15$ mas for the brightest to $\sim 30$ mas for the weakest features. The error weighted mean positions of the blue- and red-shifted masers with corresponding error bars are also indicated. The scale, assuming a distance of $11.2 \mathrm{kpc}$ is indicated in the bottom right corner.

fact that now, ten years later, the main line masers have also decreased by at least an order of magnitude (Fig. 1, right).

However, the model described above does not take the effect of maser pumping into account. As discussed in Gray et al. (2005), the response of the $1612 \mathrm{MHz} \mathrm{OH}$ maser flux could also be much more rapid if one takes into account a loss of radiative pumping efficiency after dust production has halted. In that case, a more rapid decline of mass loss can, already within a few tens of years, result in the observed exponential decay of the maser flux. The significant time-lag of at least a decade between the decay of the $1612 \mathrm{MHz}$ and the $1665 / 67 \mathrm{MHz}$ masers is however not as easily explained, as in this model the main line masers would be the first to disappear.

In the model of significantly decreasing mass loss, the $\mathrm{H}_{2} \mathrm{O}$ masers cannot be excited in the inner circumstellar envelope. This suggests that the fast $\mathrm{H}_{2} \mathrm{O}$ masers are excited in the interaction between a fast outflow and the remnant envelope as is found for water fountain nebulae. Our JVLA maser component map in Fig. 3 confirms that the red- and blue-shifted masers are separated by $\sim 50$ mas $(\sim 560 \mathrm{AU}$ at $11.2 \mathrm{kpc})$. Considering the separation, both spatially and spectrally, and elongated morphology of the red- and blue-shifted masers, we can conclude that IRAS $18455+0448$ belongs to the class of water fountain sources.

We can use the measured separation to make a crude estimate the kinematic age of IRAS 18455+0448. Assuming a constant fast outflow velocity, the age is given by

$$
\left[\frac{t_{\mathrm{kin}}}{\mathrm{yr}}\right]=4.74\left[\frac{D}{\mathrm{kpc}}\right]\left[\frac{\Delta_{\text {red-blue }}}{\text { mas }}\right]\left[\frac{\Delta V_{\mathrm{H}_{2} \mathrm{O}}}{\mathrm{km} \mathrm{s}^{-1}}\right]^{-1} \tan \left[\frac{i}{\text { degrees }}\right] \text {. }
$$

Taken from Table $1, D$ is the distance to the source, $\Delta_{\text {red-blue }}$ the red- and blue-shifted maser separation, and $\Delta V_{\mathrm{H}_{2} \mathrm{O}}$ the velocity separation. Furthermore, $i$ is the inclination from the plane of the sky. Although the distance to the IRAS $18455+0448$ is uncertain, Lewis et al. (2001) argue that the luminosity of the 

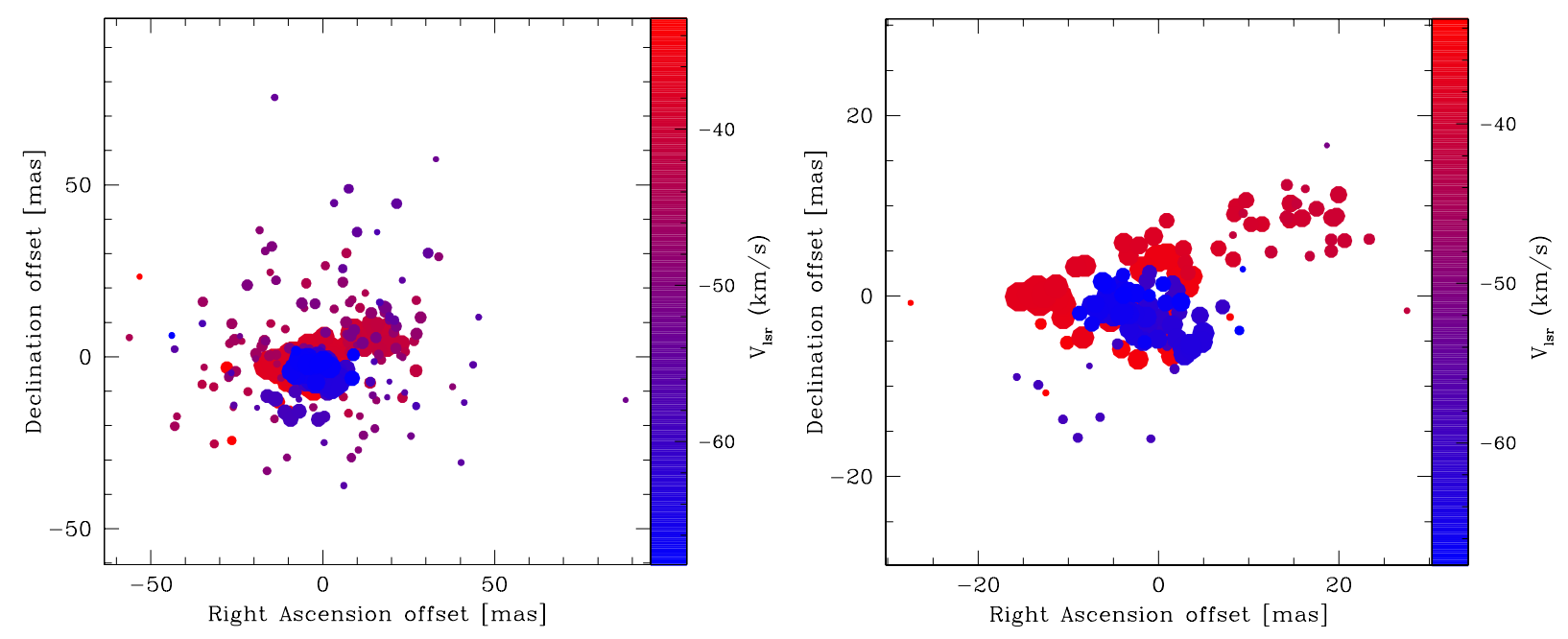

Fig. 4. Left: same as Fig. 3 for IRAS 19422+3506. All channels with a $S / N>15$ are plotted. Positional uncertainties for the individuals spots range from $\sim 2$ mas to $\sim 30$ mas for the strongest and weakest plotted masers, respectively. No average offset is detected between the red- and blue-shifted masers. Right: similar to the left but only spots with $S / N>50$ are plotted to highlight the northwest extension of the red-shifted masers. Individual errors range from $\sim 2$ mas to $\sim 10$ mas.

source suggests it is located close to the far kinematic distance of $11.2 \mathrm{kpc}$ (recalculated using the results from Reid et al. 2009) instead of the near kinematic distance $(2.1 \mathrm{kpc})$, as in that case the luminosity would be very low for a post-AGB star. Adopting $D=11.2(2.1) \mathrm{kpc}$ and an inclination of $i=45^{\circ}$, we find $t_{\text {kin }} \approx 67$ (13) yr, making IRAS $18455+0448$ one of the youngest water fountain nebulae (Imai 2007).

The rebirth of IRAS $18455+0448$ as a water fountain source, provides us with important historical information on a water fountain progenitor. Its small $\mathrm{OH}$ maser expansion velocity of $\sim 6 \mathrm{~km} \mathrm{~s}^{-1}$, implies a low main sequence mass of $\sim 1.0 \pm 0.5 M_{\odot}$ (Baud \& Habing 1983), which is noticeably lower than the masses derived for $\mathrm{CO}$ detected water fountain sources (e.g., $\mathrm{He}$ et al. 2008; Rizzo et al. 2013) and from source kinematics (Imai et al. 2012).

In the context of the model proposed by Lewis et al. (2001), the $\mathrm{OH}$ maser observations would indicate a direct connection between the events leading to a gradual decrease in mass loss, which started $\sim 1000 \mathrm{yr}$ ago, and the launch of the jet $\sim 70 \mathrm{yr}$ ago. Such a relation is not obvious if the jet originates from interaction with a binary companion during a common-envelope (CE) phase. An outflow launched as a result from CE evolution, either due to direct envelope ejection, an explosive dynamodriven jet or the formation of a circumstellar disk by shredding of the companion would act at timescales of only a few years (e.g., Nordhaus et al. 2007). The CE outflow would also not produce an obvious relation between the smooth exponential decay of $1612 \mathrm{MHz} \mathrm{OH}$ maser emission and the delayed decrease of 1665/67 MHz OH maser flux.

Instead of a gradual mass loss decrease, the main cause of the maser decay can also be, as mentioned above, the loss of pumping efficiency instead of a decrease of $\mathrm{OH}$ column density. In that case, the launch of the $\mathrm{H}_{2} \mathrm{O}$ maser jet could have occurred near simultaneously to a more rapid mass loss decrease or other event in which the dust that produces the infrared maser pump radiation is affected. In Lewis et al. (2001) it is however noted that there is no indication of any infrared variability of IRAS $18455+0448$.

\subsection{Nature of the remaining sources}

None of the other sources in our sample have a $\mathrm{H}_{2} \mathrm{O}$ maser velocity range larger than that of the $\mathrm{OH}$ masers. Should the $\mathrm{H}_{2} \mathrm{O}$ masers occur in a fast bipolar outflow, we would thus expect these to lie predominantly in the plane of the sky. However, no significant offset between the flux averaged red- and blueshifted emission peaks is seen. It is therefore more likely that the observed double peaked $\mathrm{H}_{2} \mathrm{O}$ maser spectra for these sources arise because of radial maser amplification. This indicates that the sources are high mass-loss $\mathrm{OH} / \mathrm{IR}$ stars with a $\mathrm{H}_{2} \mathrm{O}$ maser shell beyond the acceleration region, where the largest velocity coherent maser path length is, as for the $\mathrm{OH}$ masers, in the radial direction (e.g., Engels \& Lewis 1996). The multiple peaked structure, most notable in IRAS $19422+3506$, could indicate that both radial and tangential amplification is operating in an extended maser envelope that encompasses both accelerating material closer to the star and constant velocity gas farther out. Alternatively, as argued by Engels et al. (1997) for the $\mathrm{OH} / \mathrm{IR}$ star $\mathrm{OH} 39.7+1.5$, the masers occur in the same region with mode switching between tangential and radial beaming occurring in phase with the pulsation cycle and an accompanying changes in physical conditions. As shown in Fig. 4, IRAS $19422+3506$ is the only source besides IRAS $18455+0448$ that shows some structure within the $\mathrm{H}_{2} \mathrm{O}$ maser envelope at scales probed by our JVLA observations. While the overall envelope appears spherical, and there is no appreciable offset between the red- and blue-shifted emission peaks, the red-shifted emission around $-40 \mathrm{~km} \mathrm{~s}^{-1}$ is offset by $\sim 20$ mas $(\sim 200 \mathrm{AU})$ from the rest of the red-shifted masers. Monitoring the motion of such a clearly distinct maser cloud in the circumstellar AGB envelope could show if the maser cloud results from a dense clumpy mass ejection or from turbulent motions in the envelope.

\subsection{Maser variability}

The $\mathrm{H}_{2} \mathrm{O}$ masers of all sources show significant variability between different epochs. The multi-epoch Effelsberg observations covered a timescale of $\sim 500$ days. We found that the $\mathrm{H}_{2} \mathrm{O}$ masers 

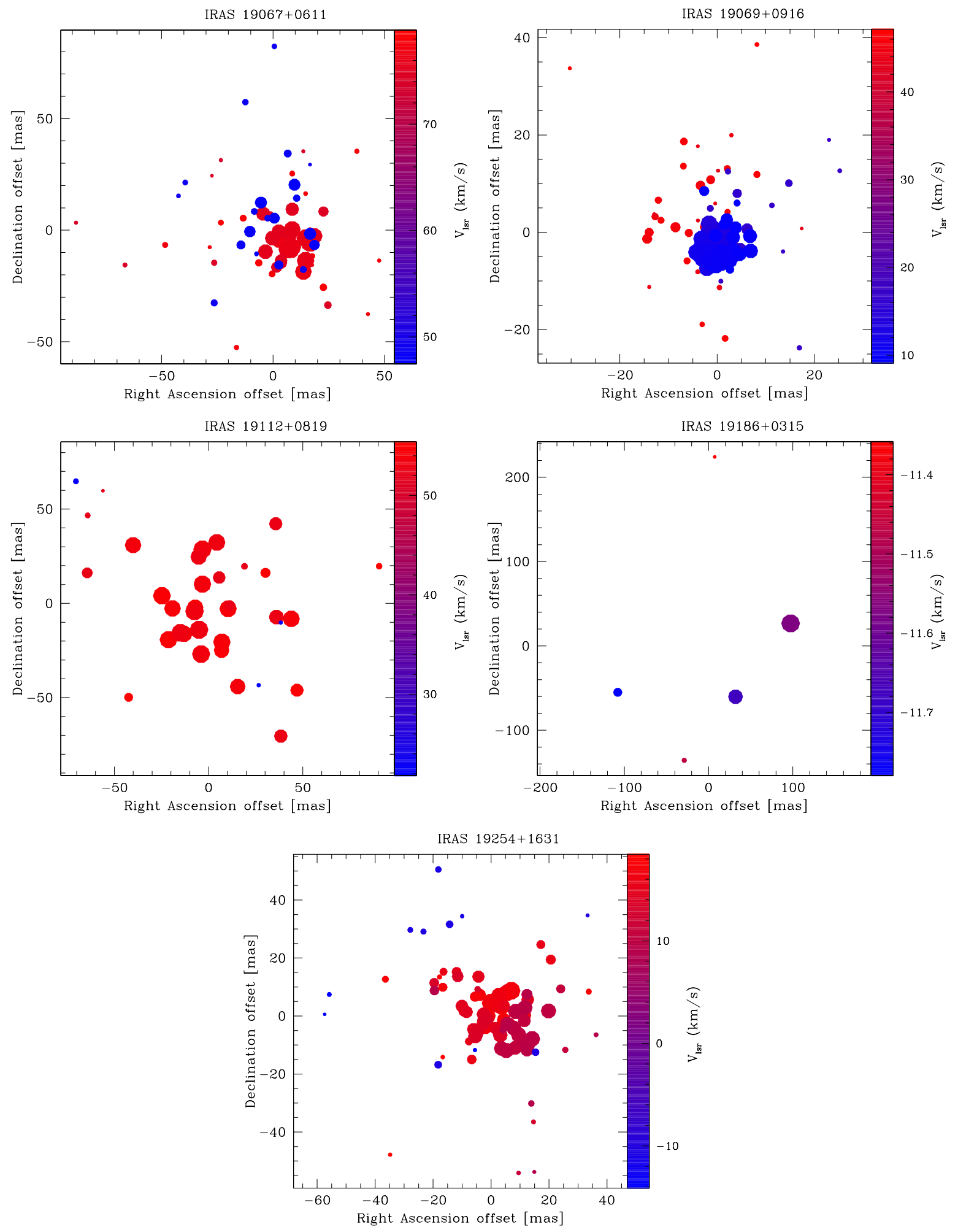

Fig. 5. Left: same as Fig. 3 for the remaining source in the sample. Depending on maser flux, different $S / N$ cutoffs are used. For IRAS 19067+0611 all channels with $S / N>5$ are plotted and error bars range from $\sim 30$ to 90 mas. For IRAS 19096+0916+0819 we use $S / N>25$ and error bars range from $\sim 5$ to 18 mas. For IRAS 19112+0819, $S / N>5$ and error bars range from $\sim 25$ to 90 mas. For IRAS $19186+0325, S / N>5$ and error bars range from $\sim 75$ to 90 mas. Finally, for IRAS $19254+1631, S / N>10$ and error bars range from $\sim 10$ to 45 mas.

exhibit significant variability in flux density and spectral characteristics. We observed the rise of new maser features that differ by as much as $35 \mathrm{~km} \mathrm{~s}^{-1}$ in velocity from features detected in the earlier observations (Engels \& Lewis 1996) for IRAS $19422+3506$. For IRAS $18455+0448$ the spectra show emission blue-shifted from the stellar velocity at $V_{\mathrm{LSR}} \approx 34.1 \mathrm{~km} \mathrm{~s}^{-1}$ between $V_{\mathrm{LSR}}=20$ and $30 \mathrm{~km} \mathrm{~s}^{-1}$ (Fig.1, left). By our second epoch in March 2011 the spectrum had changed within the same blue shifted range and a weak red-shifted feature at $\sim 46 \mathrm{~km} \mathrm{~s}^{-1}$ had appeared. One month later in April 2011, 
the most blue-shifted feature halved in flux density while the red-shifted component became stronger by a factor of a few. A further Effelsberg epoch was taken by Yung et al. (2013) in December 2011, which again showed two strong features at $\sim 19$ and $\sim 46 \mathrm{~km} \mathrm{~s}^{-1}$ and a weak third feature even more blue-shifted at $\sim 11 \mathrm{~km} \mathrm{~s}^{-1}$. Our JVLA observations from June 2013 show the now persisting red-shifted feature and show again an even more complex blue-shifted set of maser features between $\sim 13-27 \mathrm{~km} \mathrm{~s}^{-1}$. By now, the velocity spread of the $\mathrm{H}_{2} \mathrm{O}$ masers is $>38 \mathrm{~km} \mathrm{~s}^{-1}$ compared to the $12 \mathrm{~km} \mathrm{~s}^{-1}$ spread of the $\mathrm{OH}$ masers.

In Amiri (2011), it was found that the observed amount of strong variability is consistent with a variability timescale of several thousand days. This is similar to the typical $\mathrm{OH} / \mathrm{IR}$ pulsation period. For Mira variables, a similar relation between variability timescale of the $\mathrm{H}_{2} \mathrm{O}$ masers and the pulsation period was found by Shintani et al. (2008). However, since the individual sources studied in this work are mainly $\mathrm{OH} / \mathrm{IR}$ stars with periods up to $\sim 2000$ days, we do not have optical light curves available to examine whether any correlation exists between the stellar pulsation and $\mathrm{H}_{2} \mathrm{O}$ maser emission.

\section{Conclusions}

We have confirmed the previously "dead" OH/IR star IRAS $18455+0448$ to be the newest member of the class of water fountain sources. The kinematic age of IRAS $18455+0448$ is $\sim 70 \mathrm{yr}$, with the main uncertainties being the yet unknown inclination of the bipolar outflow and its distance. This is the first time that a water fountain can be directly linked to a previously studied $\mathrm{OH} / \mathrm{IR}$ star. Based on the originally low outflow velocity measured from the $\mathrm{OH}$ masers that have now disappeared, IRAS $18455+0448$ is estimated to be a fairly low-mass star, in contrast to what is generally postulated for the typical water fountain progenitor. The applicability of the expansion velocity - main sequence mass relation, which yields this low mass, to individual sources is however highly uncertain. For example observations of $\mathrm{CO}$ and its isotopologues may provide further mass estimates (e.g., He et al. 2008). Furthermore, Very Long Baseline Interferometry (VLBI) observations will be needed to derive a more accurate distance, and constrain internal kinematics. If the original model describing the approaching death of IRAS 18455+0448 (Lewis et al. 2001) is correct, the launch of the bipolar outflow in recent years is linked to a longer term slow decline of the mass loss of the water fountain progenitor. This would be difficult to reconcile with several of the binary outflow generation models that often act on relatively short timescales. However, models where the exponential decrease of the $\mathrm{OH}$ masers of IRAS $18455+0448$ is the result of a more recent disturbance of the mass loss or maser pumping mechanism that is at the same time responsible for the launch of the outflow still need to be investigated in more detail.

The remaining six sources observed with the JVLA do not show any offset between the red- and blue-shifted masers, which would be expected for a bipolar outflow not developed close to the line of sight. The double peaked nature of their $\mathrm{H}_{2} \mathrm{O}$ maser spectra is more likely the result of radial maser beaming.

Acknowledgements. W.V. acknowledges support by the Swedish Research Council (VR), Marie Curie Career Integration Grant 321691 and ERC consolidator grant 614264 . The research of N.A. was supported by the ESTRELA fellowship, the EU Framework 6 Marie Curie Early Stage Training program under contract number MEST-CT-2005-19669.

\section{References}

Amiri, N. 2011, Ph.D. Thesis, Leiden University,

https://openaccess. leidenuniv.nl/handle/1887/17981

Balick, B., \& Frank, A. 2002, ARA\&A, 40, 439

Baud, B., \& Habing, H. J. 1983, A\&A, 127, 73

Chengalur, J. N., Lewis, B. M., Eder, J., \& Terzian, Y. 1993, ApJS, 89, 189

Eder, J., Lewis, B. M., \& Terzian, Y. 1988, ApJS, 66, 183

Engels, D., \& Lewis, B. M. 1996, A\&AS, 116, 117

Engels, D., Schmid-Burgk, J., \& Walmsley, C. M. 1986, A\&A, 167, 129

Engels, D., Winnberg, A., Walmsley, C. M., \& Brand, J. 1997, A\&A, 322, 291

Garcia-Segura, G., Villaver, E., Langer, N., Yoon, S.-C., \& Manchado, A. 2014, ApJ, 783, 74

Gray, M. D., Howe, D. A., \& Lewis, B. M. 2005, MNRAS, 364, 783

He, J. H., Imai, H., Hasegawa, T. I., Campbell, S. W., \& Nakashima, J. 2008, A\&A, 488, L21

Imai, H. 2007, IAU Symp., 242, 279

Imai, H., Chong, S. N., He, J.-H., et al. 2012, PASJ, 64, 98

Kim, J., Cho, S.-H., Oh, C. S., \& Byun, D.-Y. 2010, ApJS, 188, 209

Kim, J., Cho, S.-H., \& Kim, S. J. 2013, AJ, 145, 22

Lewis, B. M. 1997, ApJS, 109, 489

Lewis, B. M., Oppenheimer, B. D., \& Daubar, I. J. 2001, ApJ, 548, L77

Likkel, L., Morris, M., \& Maddalena, R. J. 1992, A\&A, 256, 581

Martí-Vidal, I., Vlemmings, W. H. T., Muller, S., \& Casey, S. 2014, A\&A, 563, A136

Nakashima, J.-I., \& Deguchi, S. 2003, PASJ, 55, 229

Nordhaus, J., Blackman, E. G., \& Frank, A. 2007, MNRAS, 376, 599

Pérez-Sánchez, A. F., Vlemmings, W. H. T., Tafoya, D., \& Chapman, J. M. 2013, MNRAS, 436, L79

Reid, M. J., Menten, K. M., Zheng, X. W., et al. 2009, ApJ, 700, 137

Rizzo, J. R., Gómez, J. F., Miranda, L. F., et al. 2013, A\&A, 560, A82

Sahai, R., \& Trauger, J. T. 1998, AJ, 116, 1357

Shintani, M., Imai, H., Ando, K., et al. 2008, PASJ, 60, 1077

Suárez, O., Gómez, J. F., \& Miranda, L. F. 2008, ApJ, 689, 430

te Lintel Hekkert, P., Versteege-Hensel, H. A., Habing, H. J., \& Wiertz, M. 1989, A\&AS, 78, 399

Vlemmings, W. H. T., Diamond, P. J., \& Imai, H. 2006, Nature, 440, 58

Yung, B. H. K., Nakashima, J.-i., Imai, H., et al. 2011, ApJ, 741, 94

Yung, B. H. K., Nakashima, J.-i., Imai, H., et al. 2013, ApJ, 769, 20 